\title{
Visible fluorescence of biological fluids as a renal failure marker: New integrative approach
}

\author{
Artur I. Kuznetsov*, ${ }^{*}$, Aleksander Frorip*, Jekaterina Kozlova ${ }^{\dagger}$, \\ Vitali Nagirnyi ${ }^{\dagger}$, Mai Ots-Rosenberg ${ }^{\star}$, Ivo Romet ${ }^{\dagger}$ and Alar Sünter ${ }^{\S}$ \\ * Tartu Science Park, AS Ldiamon \\ Riia str. 185, 51014 Tartu, Estonia \\ ${ }^{\dagger}$ Institute of Physics, Tartu University \\ Ravila str. 14 ${ }^{\mathrm{c}}$, Tartu 50411, Estonia \\ $\$$ Department of Internal Medicine, Tartu University \\ Puusepa str. 8, Tartu 51014, Estonia \\ ${ }^{\S}$ Biomedicum, Tartu University \\ Ravila str. 19, 50411 Tartu, Estonia \\ \artur.kuznetsov@ldiamon.eu
}

Received 8 October 2014

Accepted 26 February 2015

Published 16 April 2015

\begin{abstract}
Solid phases of visible fluorescence substance (VFS) of biological fluids (blood, urine, hemodialysate) which was proposed earlier as a morbidity and mortality marker by renal failure and diabetes were investigated in-depth by the methods of electron and confocal microscopy, optical spectroscopy and matrix assisted laser desorption-ionization (MALDI) mass spectroscopy. It is shown that dry VFS exists predominantly in the form of carbon-oxygen-nitrogen $(N \approx 8.7 \mathrm{wt}$. $\%)$ nanoparticles (NPs) $(5 \leq d \leq 100 \mathrm{~nm})$. For the first time the existence of the threshold energy $E_{g} \approx 2.15 \mathrm{eV}$ for excitation of VFS was observed experimentally and confirmed by semi-empirical calculations of the bathochromic shift. A good accordance with the earlier autonomous theoretical calculations was achieved. Thus, the long wavelength limit $(575 \mathrm{~nm})$ of the spectral range where the VFS can be used as a fluorescent marker was reliably determined. A pilot MALDI comparative study of graphene oxide (GO) and urine VFS was carried out. Six kinds of nitrogenfree particles (412 $\leq M \leq 456 \mathrm{Da}$ ) were observed in each substance and possible computer models of those have been composed. It is established that along with nitrogen-containing advanced glycation end products (AGEs) also nitrogen-free carbon-oxygen-hydrogen particles (probably toxic) with the composition and structure related to GO can exist in biofluids. Both types of particles should be taken into account in search for the reasons of high mortality among end stage renal disease patients.
\end{abstract}

Keywords: Auto-fluorescence of biological fluids; AGE; glycosides; end stage renal diseases; MALDI of human metabolites; fluorescence of graphene oxide.

\Corresponding author.

This is an Open Access article published by World Scientific Publishing Company. It is distributed under the terms of the Creative Commons Attribution 3.0 (CC-BY) License. Further distribution of this work is permitted, provided the original work is properly cited. 


\section{Introduction}

Visible fluorescence substance (VFS) with the parameters $\lambda_{\text {excmax }} / \lambda_{\text {emmax }} \approx 340-350 / 430 \mathrm{~nm}$ present in biological fluids (blood, urine, hemodialysate) attracts much attention because of its clinical importance, first of all, as a marker of renal diseases. ${ }^{1-4}$ The concentration of VFS can increase very much (up to 80 times) in the serum of end stage renal diseases (ESRD) patients in comparison to the healthy controls' level. ${ }^{1,5}$ It has also been observed that the visible fluorescence (VF) intensity in hemodialysates correlates with typically high mortality index among ESRD patients and could be remarkably higher if measured in the hemodialysates of the ESRD patients who died later on. ${ }^{6}$ The same phenomenon can be traced also in the hemodialysate specific absorption measurements. ${ }^{6}$ The monitoring is going on.

Optical absorption, fluorescence and fluorescence excitation spectra of VFS are very similar to the spectra of advanced glycation end products (AGEs). ${ }^{2-4,7,8}$ Therefore, one can think that VFS is constituted to a large extent by AGEs. The AGE group is, however, very large and extremely heterogeneous whereas only a few components from this family are identified and characterized chemically as the small mass molecules, e.g., fluorescent pentosidine $\mathrm{C}_{17} \mathrm{H}_{26} \mathrm{~N}_{6} \mathrm{O}_{4} ; M=378.4$ Da. $^{2}$

High performance liquid chromatography (HPLC) and gel fractionation methods show that the abundant or sometimes dominating VFS fractions of serum and urine taken from ESRD patients and healthy controls have the molecular masses belonging to the range of, so called, middle mass molecules of $1 \sim M \leq 10 \mathrm{kDa}$ and, mostly, to the interval $1-2 \mathrm{kDa} .^{2,8-12}$

The composition, atomic structure and other properties of these fractions remain, however, unidentified. In the human metabolome database ${ }^{13}$ comprising approximately 42,000 substances present in blood and urine there are no detected molecules with the quantified concentration and masses in the range $1-10 \mathrm{kDa}$. This creates a momentum to look for ingredients other than separate molecules, which these fractions really comprise.

On this ground we have applied earlier the nanoparticles (NPs) approach to investigate the optical properties of biofluids in comparison with the properties of carbon-based NPs aqueous solutions. We have found plenty $(>10)$ of strong similarities: fluorescence spectral position, red shift and polarization of $\mathrm{VF}$, specific reaction with $\mathrm{Al}$ ions, etc. ${ }^{3,4,14}$

Nevertheless, the more precise characterization of VFS and/or NPs in biofluids still remains to be achieved. There exists an urgent requirement for such a characterization for the clinical purposes. Though very large progress has been achieved in the hemodialysis for maintenance of ESRD patients the mortality among them is "unacceptably high". ${ }^{15}$ Mortality rates among ESRD patients differ very much in the world regions extending from low $6.6 \%$ per year in Japan up to $>20 \%$ in the USA with Europe in between $13 \%$ and $15 \% \cdot{ }^{16-18}$ It seems that the blood concentrations of classical markers creatinine, urea and uric acid - are good predictors of renal failure at the early stages of disease but can give false signals at the ESRD. ${ }^{19}$ Our own data accumulated over the prolonged period of observation $(\approx 4$ years) show that by some ESRD patients' blood creatinine and urea concentrations can steadily decrease (during $\approx 2$ years) falling sometimes to the normal level shortly before a Pt's death (unpublished).

The situation with AGEs is also very complicated. Some authors doubt very much the efficacy of AGEs clearance during the hemodialysate procedures and claim the existence of the problem in a rather radical manner, e.g., "the only effective means of decreasing AGEs is renal transplantation". ${ }^{20}$

It seems, however, that one of the reasons of low clearance efficacy is that the investigations of the ESRD patients' biofluids have not been sufficiently profound and inclusive. As a result, VFS of high clinical importance is characterized poorly. For instance, there are no studies of AGEs in hemodialysates (or spent dialysates) besides our papers. ${ }^{3,4}$ To our best knowledge, the solid phases of biofluids components have not been investigated at all and, as a result, have not been characterized microscopically. The same holds in the relation to the investigation of biofluid components by the methods of the matrix-assisted laser desorption-ionisation (MALDI) mass spectrometry. Additionally, there is a great need to elucidate the mechanisms of $\mathrm{VF}$ in biofluids and to determine the region of its spectral manifestation for the reason that namely VF is directly connected with the pathology phenomena caused by the chronic renal failure. In particular, fluorescence measurements at low 
temperatures give much information about the atomic structure and optical properties of the matter under investigation. Such studies can be performed with solid phase samples, which is a novel approach to the investigation of biofluid components. It is clear now that without a systemic in-depth characterization of VFS one cannot tackle the problem of high mortality among ESRD patients.

The general aim of this study was to develop the NP approach to VFS and extend it to previously unexplored domains. Special emphasis was placed on the solid state phases of biofluids. The primary goal was to image biofluid (urine, serum) solid phases on the nanoscale in a scanning electron microscope (SEM) and to carry out the elemental analysis of NPs in situ. An additional goal in the microscopic domain was the exploration of biofluid solid state pieces with a confocal microscope to get information about the VF topography in connection with the phenomenon of the red shift of VF.

Secondly, the study was aimed at a precise measurement of VF spectra of biofluid solid phases at room and liquid nitrogen temperatures (RT and LNT) and at the comparison of these spectra with the spectra of carbon-based NPs in water solutions and in dry layers. Modeling the processes and deducing important parameters such as the red shift gradient in $\mathrm{VF}$ and the value of the energy gap $E_{g}$ was planned as a conclusive achievement in the fluorescence domain. The final goal in the optical part of the given study was to determine the spectral region where the VFS can be used for practical purposes.

The third group of experiments was motivated by the earlier revealed ${ }^{3}$ similarity in the VF and UV spectra in biofluids and aqueous graphene oxide (GO) solutions. Such a heuristic comparison was extended to mass spectrometry performed by the MALDI method to get insight into the atomic structure of the middle mass components of biofluids by using the "parallel" information on GO.

\section{Samples and Methods}

Dry samples were prepared from urine taken from the healthy controls and from ESRD patients' blood. Blood and hemodialysate samples were collected at the hemodialysis department of the Tartu University Hospital.
Biofluids were fractionated in gel columns PD-10 (Sephadex $^{\text {TM }}$ G-25) with the cut-off $M<5000$ Da for the removal of proteins. For further experiments, the middle mass fractions $(M<5000 \mathrm{Da})$ eluted next to protein fractions were used. Vitamin $\mathrm{B}_{12}(M=1355 \mathrm{Da})$ was added into the starting fluids for the control of mass distribution among fractions. The control of $\mathrm{B}_{12}$ distribution and concentration estimation was done at a $\mathrm{LC} / \mathrm{MS}$ unit Aqilent Technologies 6540 UHD. ${ }^{3}$ The dominant presence of $\mathrm{B}_{12}$ in the first post-protein, i.e., middle mass fraction, was established. It should be mentioned that the urine taken from healthy controls does not normally contain much proteins. Therefore, the urine fractionation was undertaken to get rid of any traces of proteins and large peptides. In the further text, "urine fraction" will stand for this middle mass fraction if not noted otherwise. A biochemical analysis (Cobas 6000 Roche) has shown that important metabolites, urea and creatinine, in concentrations of $\sim 10 \mathrm{mmol} / \mathrm{L}$ and $\sim 1 \mathrm{mmol} / \mathrm{L}$, respectively, and the traces of uric acid were also present in these urine fractions.

To prepare a solid state phase, the urine fraction liquid was dropped onto a gold substrate and dried in hot air for tens of seconds. The samples prepared this way were examined with a SEM Helios NanoLab ${ }^{\text {TM }}$ (FEI Company) operating in the standard mode.

Another part of the same urine fractions was lyophilized (cold drying) and the VFS powder, very hydrophilic, was obtained. The larger pieces of this powder were placed into a confocal microscope Zeiss LSM710 operating with four lasers and filters at wavelengths $405 \mathrm{~nm}$ (filter $410-483 \mathrm{~nm}$ ), 488 (494$571), 561$ (575-712) and $633 \mathrm{~nm}(642-705) \mathrm{nm}$. To avoid intensity saturation (overexposure), minor laser intensities were used. These were set at 0.15 , $0.31,0.08$ and $0.15 \mathrm{~mW}$, respectively. The objective magnification was $20 \times$ in all cases. The original software of the confocal microscope was used for image processing.

For fluorescence studies at different temperatures, the VFS powder was mounted into a liquid nitrogen cryostat (Janis VPF-800) and VF measurements were carried out in the spectral range 220-700 $\mathrm{nm}$ at temperatures $77-295 \mathrm{~K}$. For the VF measurements of biofluids at room temperature they were filled into quartz cuvettes and placed into a multi-purpose dark box. The VF of a sample was excited by a $400-\mathrm{W}$ deuterium lamp DDS-400 
through a double-quartz prism monochromator DMR-4. Emission spectra were recorded with a SpectraPro-2300i (Acton Research Corporation) grating monochromator or with another DMR-4 monochromator, each equipped with a Hamamatsu photon counting head H8259-02. In the case of the grating monochromator, an additional yellow bandpass glass filter ZhS-4 (transparent from $400 \mathrm{~nm}$ to infrared) was placed in front of the entrance slit to cut a possible second order of the excitation light. A Spellman XLF device $(50 \mathrm{kV}, 0.15 \mathrm{~mA})$ with a dose rate $32 \mathrm{~Gy}$ (Gray) $/ \mathrm{s} / \mathrm{cm}^{2}$ was used for the X-ray irradiation of powder samples. Data acquisition was performed with the aid of the LabView-based software.

The MALDI experiments were performed with a standard Voyager De PRO Applied Biosystems unit with sample irradiation by laser light at $337 \mathrm{~nm}$. Three matrixes $\left(2^{\prime}, 4^{\prime}, 6^{\prime}\right.$-Trihydroxyacetophenone monohydrate (THAP), 2.5-dihydroxy-bensidine (DHB) and $\alpha$-Cyano-4-hydroxycinnanic acid (CHCA) (CHCA was applied only with GO) were used. For the MALDI experiments the middle mass urine fraction of the healthy controls, prepared in the PD-10 columns as described above and concentrated afterward, was fractionated additionally to receive narrow fractions. This was achieved with the use of a HPLC unit ÄKTA Purifier 900 (GE Healthcare) and a column Jupiter $5 \mu$ C18 $300 \mathrm{~A}$ $250 \times 21 \mathrm{~mm}$. Three main fractions with elution times 8.5 (most hydrophilic), 9 and $18 \mathrm{~min}$ (most hydrophobic) were extracted, separated and collected using the optical absorption detection at the wavelengths 238, 290 and $330 \mathrm{~nm}$ (see Electronic Supplementary Information, ESI Fig. 1). The absorption at $330 \mathrm{~nm}$ was detectable and could be used only for the fraction $8.5 \mathrm{~min}$. We applied the standard dried droplet method used usually in the MALDI measurements. The prepared urine fractions were diluted in the ratio 1:1 with one of saturated matrixes, e.g., THAP, in the solution containing $50 \%$ acetonitrile and $0.1 \%$ trifluoroacetic acid (TFA). This mixture was dropped onto the standard multisample plate and dried for about $20 \mathrm{~min}$ in open air before measuring. The processing of the recorded mass spectra was performed with the original software of the MALDI instrument and standard Excel Office 2013. The modeling of atomic structure of the MALDI products was carried out with the use of a freely available Avogadro software.
Healthy controls' urine and GO were used in the comparative MALDI experiments. GO was the same as that used in our previous study. ${ }^{3}$ The stoichiometric ratio $\mathrm{C}$ :O was 2.5 in this GO. The presence of hydrogen was not quantified. Oxygen is present in GO usually in different forms of the epoxy $(-\mathrm{C}-\mathrm{O}-\mathrm{C}-)$ as well as $-\mathrm{OH},-\mathrm{COH}$ and $-\mathrm{COOH}$ groups, $\mathrm{C}=\mathrm{O}$ bond and heterocyclic position in the six-member rigs. The water solution (suspension) of GO contained one-sheet flakes with the thickness $h \leq 1 \mathrm{~nm}$ and lateral dimensions of 50-100 nm. The suspension exhibited VF and UV fluorescence spectra much alike the spectra of biofluids both in undoped state and after doping with $\mathrm{Al}$ ions. ${ }^{3}$ The GO solid state samples were prepared for the MALDI measurements in the same way as it was described above for urine.

\section{Results}

\subsection{SEM images and elemental analysis}

One of the SEM images (the magnification $100000 \times$ ) is represented in Fig. 1(a). The particles of very different sizes scaling from $\sim 100 \mathrm{~nm}$ and more to the smallest ones of $\leq 7 \mathrm{~nm}$ are seen. One can also observe a rather picturesque agglomeration of NPs into aggregates of different forms and submicron dimensions. The NPs smaller than $5 \mathrm{~nm}$ could not be observed in this SEM examination. The insert in Fig. 1(a) demonstrates a magnified image of a NP with the diameter $7.5 \mathrm{~nm}$.

In the other image $(250000 \times)$, a large space around the marked NP of $d=103.8 \mathrm{~nm}$ is empty of any NPs (Fig. 1(b)). This can be a result of the Ostwald ripening phenomenon leading to the absorption of smaller NPs by the larger ones. ${ }^{21}$

The elemental analysis was done in situ making use of larger particles. A typical result is shown as the characteristics emission spectrum in Fig. 2. Five peaks of metals (obviously dominant gold $\mathrm{Au}$ (93 wt.\%) at $2.15 \mathrm{keV}$ with two smaller Au satellites at 1.65 and $2.4 \mathrm{keV}$, sodium $\mathrm{Na}(1.05 \mathrm{keV})$ and, unexpectedly, aluminum $\mathrm{Al}(1.5 \mathrm{keV})$ were observed in parallel to the peaks of carbon $\mathrm{C}$ (1.9 wt.\%, $0.24 \mathrm{keV})$ and oxygen $\mathrm{O}(3.9 \mathrm{wt} . \%, 0.52 \mathrm{keV})$ with the minor hump of nitrogen $\mathrm{N}(0.55 \mathrm{wt} . \%, 0.4 \mathrm{keV})$ in-between. We observed the NPs with a similar composition in a lyophilized sample of the ESRD patients' serum as well. 


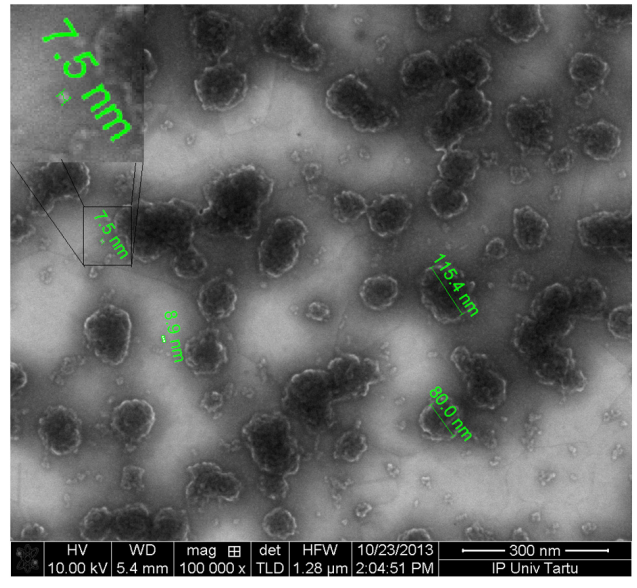

(a)

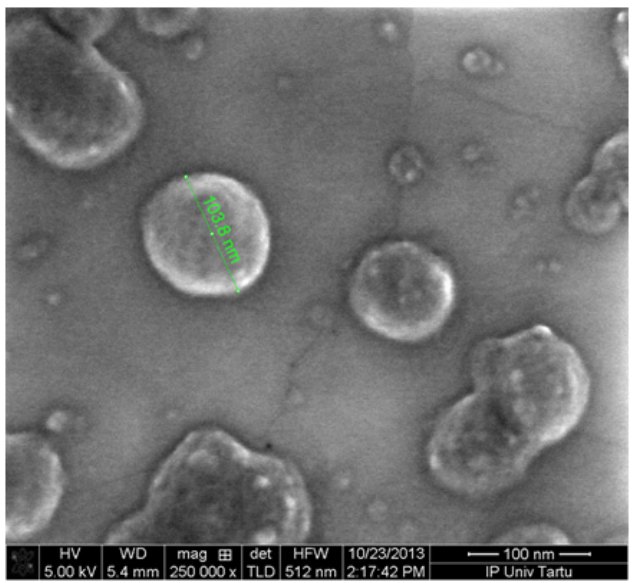

(b)

Fig. 1. SEM images of the VFS particles on a thin gold substrate with magnification $100000 \times$ (a) and $250000 \times$ (b). Inset in Fig. 1 (a) demonstrates a magnified separate $\mathrm{NP}$ of the diameter $7.5 \mathrm{~nm}$.

\subsection{Confocal microscope images}

Figures 3(a)-3(f) show six images (objective magnification $20 \times$ ) of a piece of dry urine fraction under different laser excitations ( $a, b, c, d)$ taken in the sequential mode with the appropriate filters, white field image (e) and a merge of colors in Fig. 3(f). The glow patterns in three colors - blue [Fig. 3(a); excitation $405 \mathrm{~nm}$, filter 410-483 nm], green [Fig. 3(b); $488 \mathrm{~nm}$ and $494-571 \mathrm{~nm}$ ] and red [Fig. 3(c); $561 \mathrm{~nm}$ and $575-712 \mathrm{~nm}$ ] — manifest an equally uniform distribution of emitting centers in the most part of the sample volume (some minor non-fluorescent inclusions of unknown origin are also seen). Figure 3(e) in the black-white contrast demonstrates the lateral dimensions of the glowing subject $\left(l_{\max } \sim 500 \mu \mathrm{m}\right)$ whereas Fig. 3(f) is the result of the three main colors merge into the almost white image. This demonstrates that a fair color balance can be achieved under these excitation conditions.

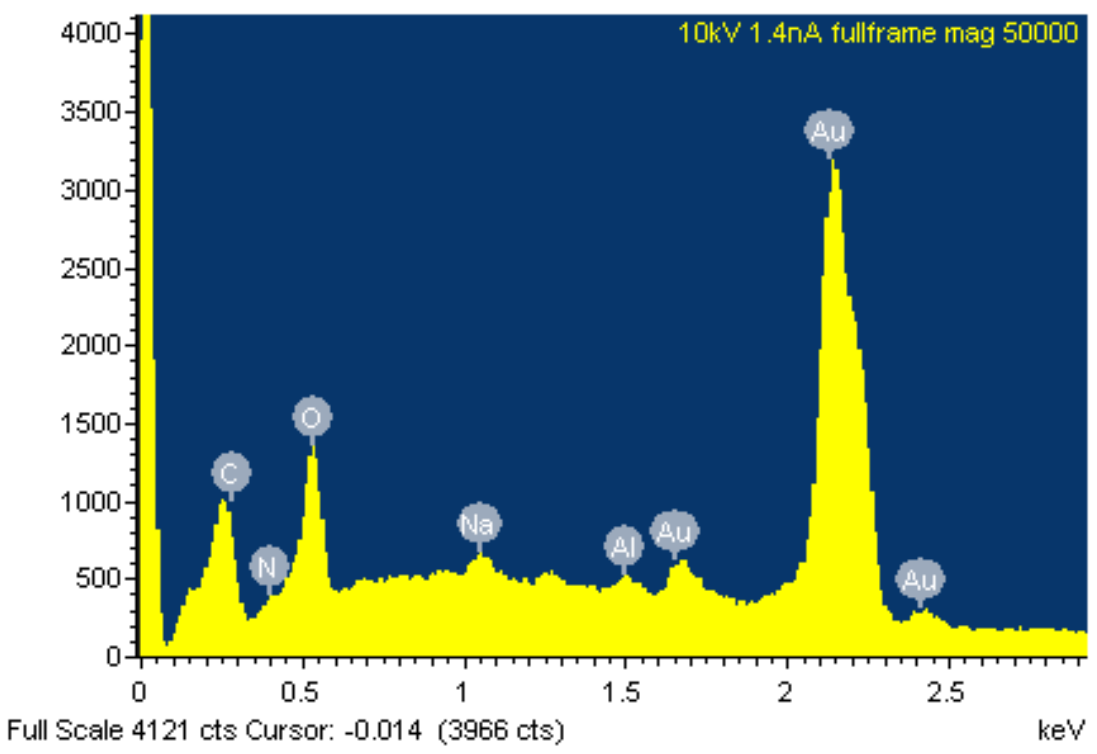

Fig. 2. Characteristics emission of the elements abundant in the urine NPs under excitation by electron beam $10 \mathrm{kV}, 1.4 \mathrm{nA}$. The $x$-axis represents the energies of emitted photons from 0.1 to $2.8 \mathrm{keV}$ with the specified peaks at 0.24 (carbon $\mathrm{C}$ ), 0.4 (nitrogen $\mathrm{N}$ ), 0.52 (oxygen $\mathrm{O}$ ), 1.05 (sodium Na), 1.5 (aluminum Al) and three peaks at 1.65, 2.15 and $2.4 \mathrm{keV}$ of the gold Au. The $y$-axis represents the intensity of emission in linear arbitrary unites. Note that the line amplitudes do not determine directly the elements concentrations measured in the weight $\%$. 


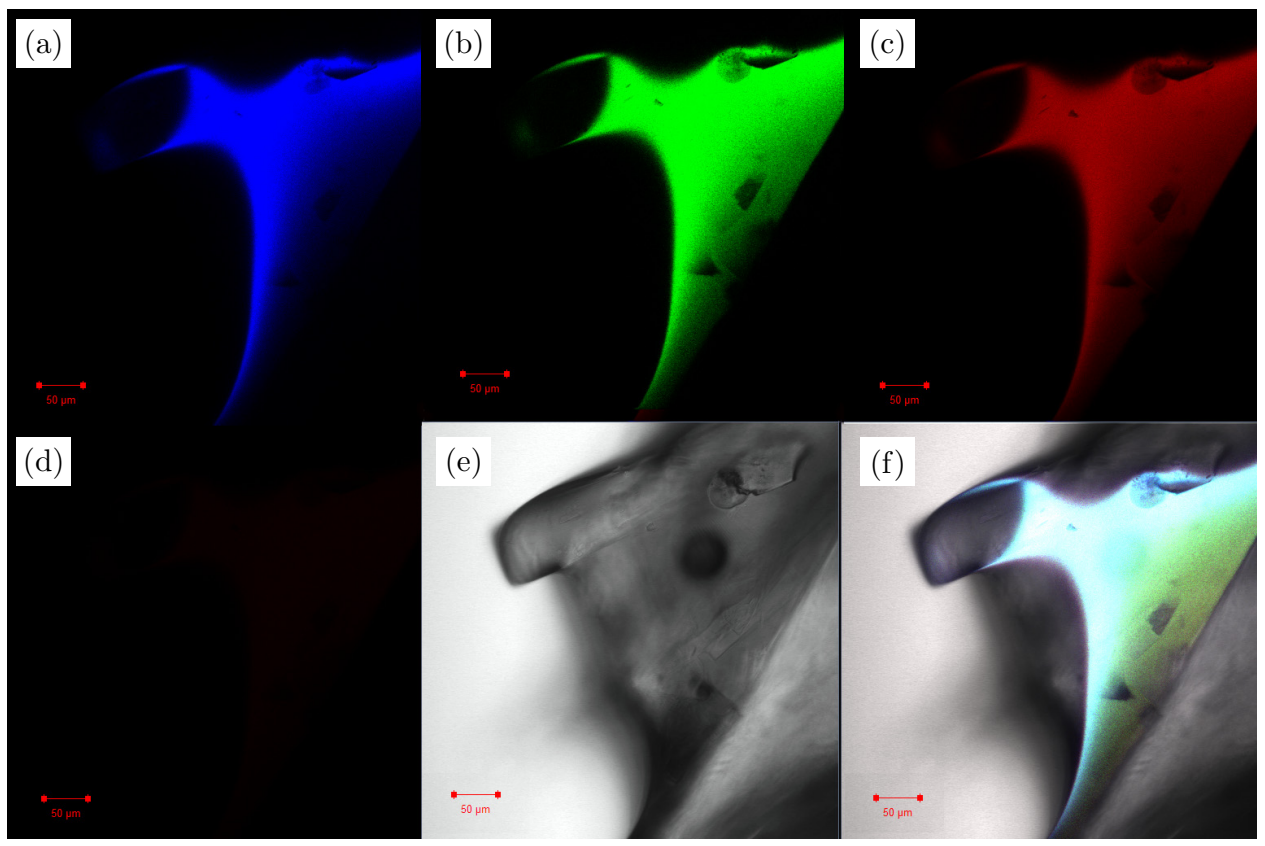

Fig. 3. A piece of lyophilized urine fraction in a confocal microscope (objective magnification $20 \times$ ). Excitation wavelengths and bandpass filters used here are: (a) $405 \mathrm{~nm}(410-483 \mathrm{~nm})$ (blue); (b) $488 \mathrm{~nm}$ (494-571 nm) (green); (c) $561 \mathrm{~nm}(575-712 \mathrm{~nm})($ red); (d) $633 \mathrm{~nm}(642-705 \mathrm{~nm})$ ("no-image"); (e) white-black image and (f) merge of three colors from a, b, c.

The "no-image" Fig. 3(d) where only very weak sample silhouette can be seen as a result of the filter residual transmittance of the exciting light is of special significance. In this case, the excitation with the red laser $633 \mathrm{~nm}$ (photon energy $1.96 \mathrm{eV}$ ) was performed. This was not followed by emission under the excitation power $0.15 \mathrm{~mW}$ which is twice as high as in the case of near energy green excitation at $561 \mathrm{~nm}(2.21 \mathrm{eV})$ in Fig. 3(c). This result is a convincing manifestation of the existing threshold excitation energy in the VFS. Evidently, this threshold energy (or the energy gap $E_{g}$, see below) has some intermediate value in the range $1.96-2.21 \mathrm{eV}$. The $E_{g}$ value determines the longwavelength limit of the spectral range where the VFS can be used as a fluorescent marker. An additional confirmative estimation of the energy gap $E_{g}$ can be made on the basis of VF measurements data.

\subsection{Fluorescence spectra and red shift}

Two fluorescence spectra measured with a dry fractionated urine sample and a hemodialysate liquid sample both under excitation $370 \mathrm{~nm}$ at RT are compared in Fig. 4. One can see that the coincidence of the spectra is good implying a common nature of VFS in both samples (see also Ref. 4).
Earlier, we have observed a good overlapping of emission spectra of hemodialysate and sugar derived NPs in water. ${ }^{3}$ One can take the given facts as an indication to the strong similarity in the nature of fluorescent entities in such apparently very different substances as liquid and dried biofluids as well as artificial carbon-based NPs aqueous solutions.

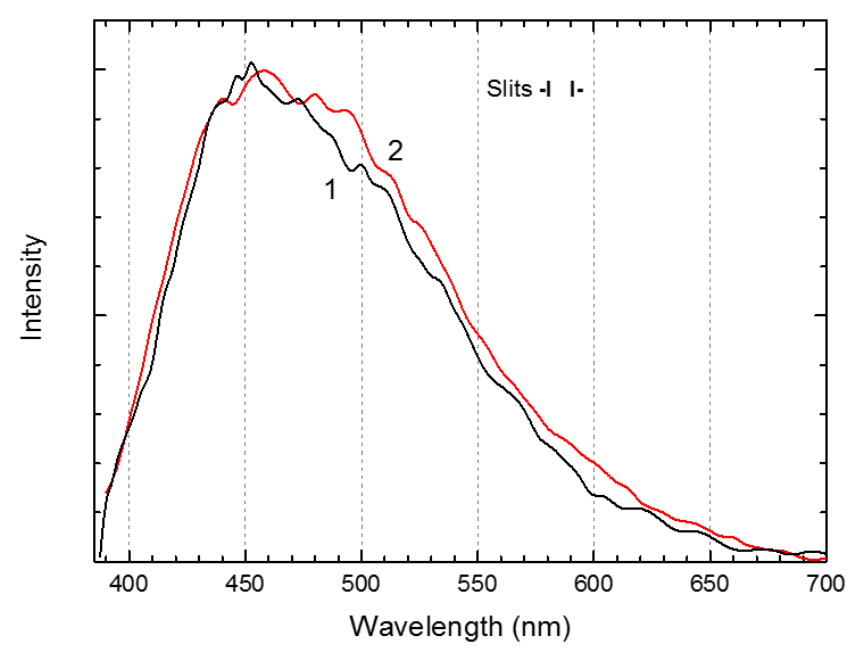

Fig. 4. Fluorescence spectra of hemodialysate (1) and urine fraction powder (2) at excitation $370 \mathrm{~nm}$ at $T=295 \mathrm{~K}$. An average spectral width of the monochromator slits is shown by brackets. 


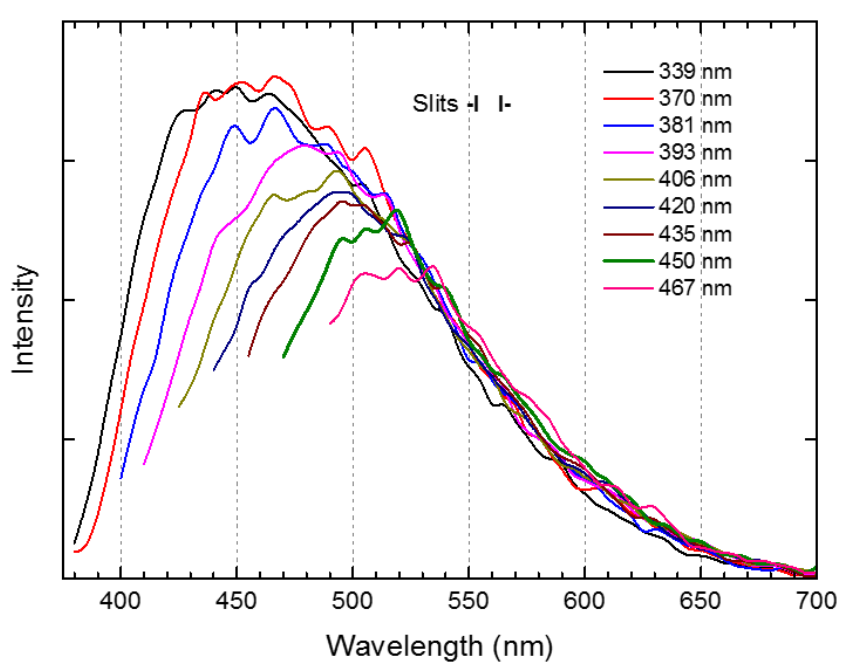

Fig. 5. Fluorescence bands of urine fraction powder at various excitation wavelengths at $77 \mathrm{~K}$. An average spectral width of the monochromator slits is shown by brackets.

Figure 5 represents the emission spectra of the dry VFS powder of a urine fraction measured at $T=77 \mathrm{~K}$ under different excitations of equal intensity in the spectral range $339-467 \mathrm{~nm}$ (3.65$2.65 \mathrm{eV})$. The low-temperature fluorescence spectra of dry metabolites extracted from urine are measured, to our knowledge, for the first time. The fluorescence band maxima move in the long wavelength direction upon the decrease of the excitation energy, i.e., they exhibit the bathochromic or red shift. An analogous red shift occurs at room temperature. This is the same red shift as that described by us earlier in the fluorescence of real biofluids. ${ }^{14}$ The analogous red shift was also observed many times in the aqueous solutions of carbon-based $\mathrm{NPs}_{\mathrm{s}}{ }^{22}$ including GO. ${ }^{23,24}$ A remarkably similar red shift is one of the most important phenomena which unites both classes of condensed matter - the biofluids and aqueous solutions of carbon NPs.

The red shift seen in Fig. 5 can be described by the linear equation in the energy terms as $y=a x+b$, where $y$ is the emission and $x$-excitation photon energy in eV. For the spectra measured at LNT as in Fig. 5 we have obtained the equation:

$$
y=0.49 x+1.08 \mathrm{eV} .
$$

For RT the dependence has a very similar form: $y=0.46 x+1.15 \mathrm{eV}$. This is because the temperature effects are very weak or not observable at all. The normalized fluorescence spectra measured at 295 and $77 \mathrm{~K}\left(\lambda_{\text {exc }}=354 \mathrm{~nm}\right)$ are shown in Fig. 6 .

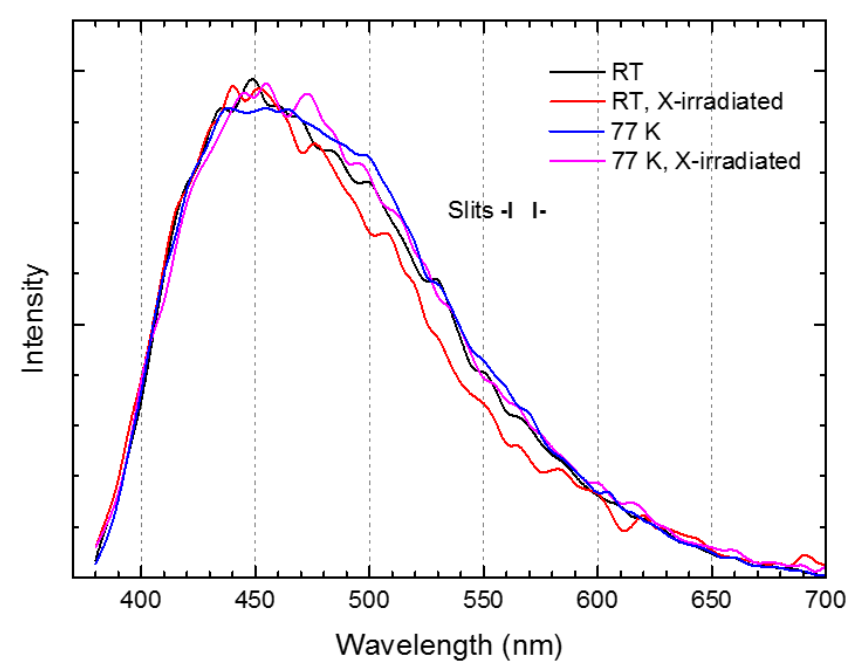

Fig. 6. Fluorescence spectra of urine fraction powder at room temperature (RT) and $77 \mathrm{~K}$ of a sample before and after irradiation by X-rays at RT to the dose of $57.5 \mathrm{kGy}$. An average spectral width of the monochromator slits is shown by brackets.

One can see that the spectra coincide with each other perfectly. Only relatively small increase in intensity (25-30\%) could be observed by lowering temperature from 295 to $77 \mathrm{~K}$.

Interestingly, X-irradiation with a high dose of $57.5 \mathrm{kGy}$ does not affect the emission properties of VFS at RT and LNT (Fig. 6). This fact manifests a high irradiation resistivity of the VFS and points to its stable structure.

\subsection{Matrix assisted laser desorption- ionization mass spectra}

Urine MALDI spectra were sufficiently intense only in the range of the values $M / Z \leq 600 \mathrm{Da}$ where $M$ is the mass and $Z$ is charge of a particle (see ESI Fig. 2). In the region $M / Z<400$ Da spurious peaks belonging to the used matrixes can appear in the spectra. ${ }^{25}$ To avoid these possible false signals we concentrated our attention mainly on the region 400-500 Da. Two fragments of the spectra (THAP matrix) with the lines needed for further analysis for GO and for the urine most hydrophilic fraction $8.5 \mathrm{~min}$ are represented in the region $M / Z 410$ $460 \mathrm{Da}$ in Fig. 7. Other possible combinations of urine fractions and matrixes were also used in the measurements and analysis. In almost all cases, we detected for both substances three lines in this region with approximate $M / Z$ masses 413,429 and $441 \mathrm{Da}$. Other lines 425 and $437 \mathrm{Da}$ appeared mostly 


\section{A. I. Kuznetsov et al.}

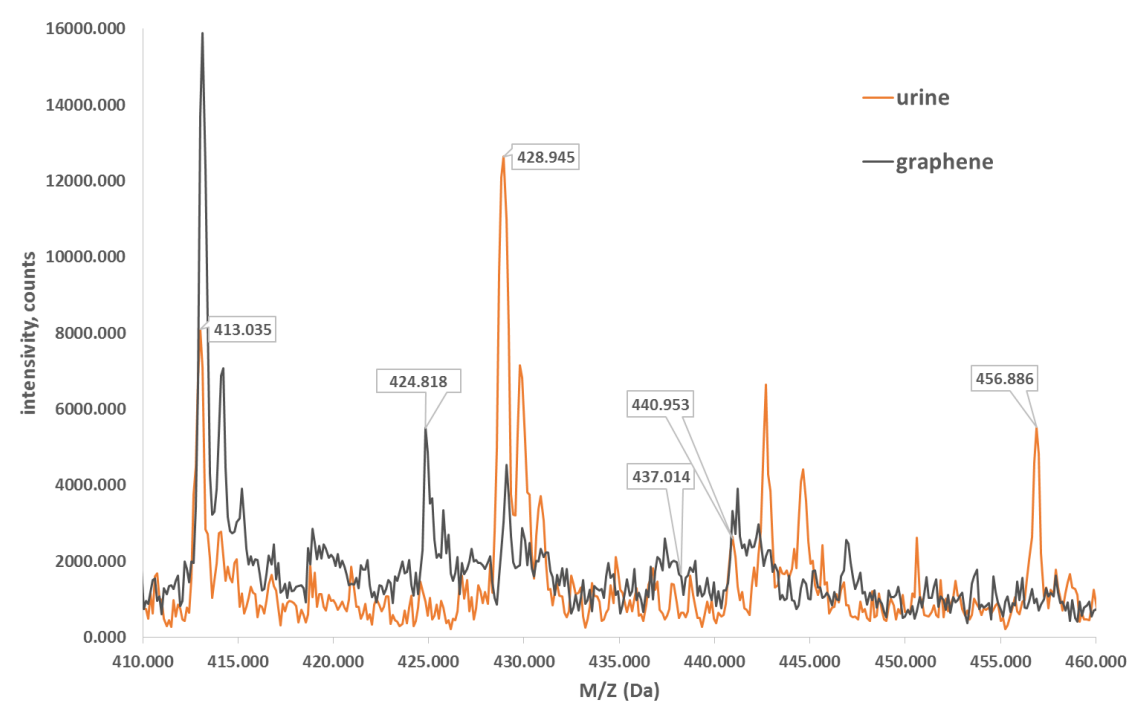

Fig. 7. Fragments of MALDI spectra (matrix THAP) of GO and urine fraction 8.5 min in the region $M / Z 410-460 \mathrm{Da}$.

in the GO spectra, while the line $457 \mathrm{Da}$ is observed mainly in urine spectra. In Table 1, we averaged the weight numbers for these lines in GO on the basis of our numerous measurements. For comparison, we show also similar line parameters deduced from figures in the study of carboxylated magnetic graphene $^{25}$ and averaged by us. One can see that the mass values coincide fairly well in both series. Almost all main MALDI lines in Table 1 are accompanied by weaker ones with larger masses and equidistant intervals of $1 \mathrm{Da}$. These multiplets are analyzed below.

One can see that the lines in Fig. 7 and Table 1 are separated from the line 1 (413 Da) by certain intervals equivalent to the combinations of the atomic masses of carbon and/or oxygen. The spacing between the lines 1 and 2 is $12 \mathrm{Da}$ or one carbon $\mathrm{C}$ atom mass; between 1 and 3-16 Da or one oxygen $\mathrm{O}$ atom; between 1 and $4-24 \mathrm{Da}$ or two $\mathrm{C}$ atoms; between 1 and $5-28 \mathrm{Da}$ or $\mathrm{C}+\mathrm{O}(\mathrm{CO})$ atoms and between 1 and 6-44 Da or $\mathrm{C}+2 \mathrm{O}(\mathrm{COO})$ atoms. All these atoms, single $\mathrm{O}$ atoms or their combinations with hydrogen $(\mathrm{OH}, \mathrm{COH}$ and $\mathrm{COOH})$, are wellknown functional groups in the GO structure (see Sec. 2). These facts allow us to consider the particle 413.24 $\mathrm{Da}$ inducing the line 1 as a core particle (further we will call it Core) from which the larger mass particles can be derived or composed.

The averaged parameters for the MALDI lines obtained for urine are given in Table 2. It is remarkable, that the same lines 1-6 are observed in the urine and GO spectra. Lines 2 and 4 were always weak with a rather occasional appearance in both GO and urine spectra. Line 6 (457.024 Da) could be strong in the urine spectra. Line 3 (429.099 Da) could dominate not only the limited range of $410-460 \mathrm{Da}$ as in Fig. 7 but also the urine spectra measured in a wider interval $(M / Z=200$ $550 \mathrm{Da})$. None of the lines given in Tables 1 and 2 were detected in the control spectrum taken with carboxylfluoroscein (the standard $M$ is $376.32 \mathrm{Da}$ ). Only the main line at 377.18 and a satellite at 378.21 Da were observed.

A strong similarity between GO and urine spectra can also be seen in the satellite lines sequences observed at the larger mass sides from the main lines. In Fig. 8, we demonstrate such a three-satellite pattern for the most intense line 3 of urine $(428.945 \mathrm{Da})$. All lines have the fine structure caused by multichannel registration. Whatever the reason of the appearance of satellites (the presence of the isotope $\mathrm{C}^{13}$ with abundance of $1.109 \%$ or a

Table 1. Lines (quantified in Da) in MALDI spectra of graphene oxide.

\begin{tabular}{lcccccc}
\hline Source & Line 1 & Line 2 & Line 3 & Line 4 & Line 5 & Line 6 \\
\hline This study & $413.345 \pm 0.229$ & $424.879 \pm 0.057$ & $429.267 \pm 0.173$ & $437.144 \pm 0.152$ & $441.375 \pm 0.237$ & 457.328 \\
Ref. 25 & $413.139 \pm 0.011$ & & 429.175 & $437.12 \pm 0.04$ & $441.25 \pm 0.019$ & \\
\hline
\end{tabular}


Table 2. Lines with averaged values in Da in MALDI spectra of urine fractions.

\begin{tabular}{lccccc}
\hline Line 1 & Line 2 & Line 3 & Line 4 & Line 5 & Line 6 \\
\hline $413.12 \pm 0.101$ & $425.1 \pm ?$ & $429.099 \pm 0.126$ & $437.14 \pm 0.152$ & $441.138 \pm 0.154$ & $457.024 \pm 0.122$ \\
\hline
\end{tabular}

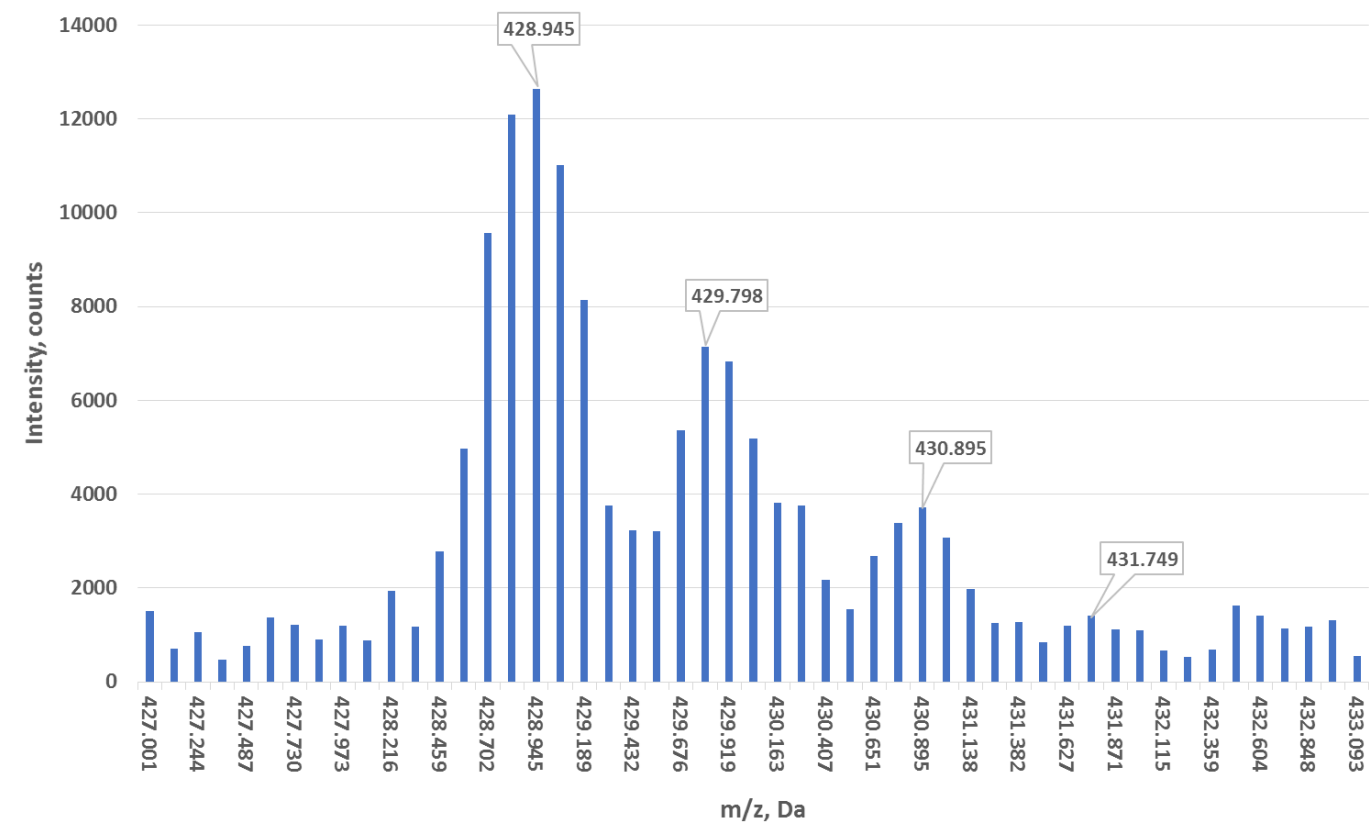

Fig. 8. The structured main line $M / Z=428.945 \mathrm{Da}$ in the MALDI spectrum of the urine fraction 8.5 min elution accompanied by three equidistant satellites at $429.798,430.895$ and 431.748 Da.

change in the number of hydrogen atoms connected to the given particle) the strictly envisaged interval of 1 Da must be observed and can be used for the check of technical accuracy in the measurements. We have made use of all data available from multiple measurements and obtained the average interval value $1.01595 \pm 0.06781 \mathrm{Da}$. Thus, we see that the technical accuracy is sufficient to distinguish or/and confirm identity of lines in GO and urine spectra.

\section{Discussion}

We have observed earlier the presence of NPs in protein-free fractions of liquid urine by the methods of small angle X-rays scattering (SAXS) and dynamical light scattering (DLS). ${ }^{3}$ In both cases, the existence of nanoentities with the diameter lying in the range $4-6 \mathrm{~nm}$ was revealed. In all probability, the NPs detected earlier by the SAXS and DLS methods in the liquid medium and the NPs with $d \approx 5-7 \mathrm{~nm}$ observed in the solid phase by SEM in the present work are of the same origin and composition. Examination with the use of a high resolution transmission electron microscope (TEM) can, in principle, gives insight into the atomic structure of such metabolic NPs derived from biofluids.

A prominent tendency of NPs to aggregate into larger complexes clearly seen in Figs. 1(a) and 1(b) can be considered as the final stage of the NPs conglutination occurring at very high concentrations during the drying process and water evaporation in the course of sample preparation. One can think that the most probable driving aggregation mechanism is the Ostwald ripening. Indeed, the SEM images in Fig. 1(a) and 1(b) are very much like the classical (tutorial) pictures illustrating this phenomenon (see ESI, Sec. 2).

We have earlier found some specific effects in the VF spectra of urine and serum, which were induced only by the dilution of biofluids, i.e., by association/ dissociation processes. ${ }^{4}$ These processes of association/dissociation can evidently lead to the emergence of NPs of diverse and complex forms. This conclusion is supported also by the recently revealed 
polarization of $\mathrm{VF}^{3,26}$ Otherwise, the polarization phenomenon could not exist for small mass molecules in liquids. Our control experiments carried out with the metabolite 4-Pyridoxic acid solutions $\left(\mathrm{C}_{8} \mathrm{H}_{9} \mathrm{NO}_{4}, \quad M \approx 183 \mathrm{Da}\right)$ have confirmed this conclusion.

It should be mentioned that we have observed also the VF polarization of sugar derived carbon NPs. ${ }^{3}$ This fact substantiates once more the similarity between biofluids and carbon NPs aqueous solutions in relation to the existence of fluorescent bio-NPs.

The ratio of carbon and oxygen in our NPs observed with a SEM and, namely, the prevalence of oxygen over carbon by a factor of 2 is an important feature. It can be compared with the data known for carbon-oxygen nanodots. For instance, in the nanodots done from candle soot, the ratio was $\mathrm{C} / \mathrm{O}=36.8: 44.7 \%$ or $1: 1.2$, i.e., oxygen was excessive in this composition. ${ }^{22}$ The carbon dots characterized by the red shift have an enhanced surface oxidation level as a result of massive presence of hydroxyl $\mathrm{OH}$ and carboxyl $\mathrm{COOH}$ groups on the surface. ${ }^{27}$

Relatively low content of nitrogen ( $0.55 \mathrm{wt} . \%)$ is also of great importance since it clearly shows that the matter constituting our NPs is other than urea $\mathrm{CH}_{4} \mathrm{~N}_{2} \mathrm{O}$ or creatinine $\mathrm{C}_{4} \mathrm{~N}_{7} \mathrm{H}_{3} \mathrm{O}$, where nitrogen is a dominant component (43 and $59 \mathrm{wt} . \%$, respectively). On the other hand, AGE as a product of reaction of proteins with sugar ${ }^{28}$ cannot be very rich in nitrogen since the mean nitrogen percentage is $\approx 16 . \mathrm{wt} \%$ in proteins and $0 \%$ in sugar. In our determination of elements, nitrogen has $8.7 \mathrm{wt} . \%$ of the triple mass $\mathrm{C}+\mathrm{O}+\mathrm{N}$ that meets the assumption that the NPs studied can be AGE-based particles. Interestingly, the nitrogen content is $8.6 \mathrm{wt} . \%$ in the Amadori products which are derived from $\mathrm{N}$-glycosides and can serve as an intermediate material for the formation of AGEs. ${ }^{29}$

The Eq. (1) for the red shift can be used for the estimation of minimal energy gap $E_{g}$ of VFS in two slightly different but self-consistent ways. If we suppose $y=x$ in Eq. (1), it would mean the excitation and emission energies are in resonance and the Stokes shift energy is zero. This purely theoretical approach gives the resonance value or $E_{g} \approx 2.12 \mathrm{eV}$ for both RT and LNT cases.

We obtain the close value if the Stokes shift is determined from the measured spectra drawn in
Fig. 5. We get equation:

$$
z=0.56 x-1.23 \mathrm{eV},
$$

where $z$ is the Stokes shift and $x$-excitation energy in $\mathrm{eV}$. A resonance $(z=0)$ is situated at the excitation energy $x=2.19 \mathrm{eV}$, which is, in essence, also the $E_{g}$ value.

More realistic is, however, to assume that the Stokes shift is not negligible. If we let the Stokes shift to be $<0.1 \mathrm{eV}$, e.g., $0.05 \mathrm{eV}$, which is a quite reasonable value for organic molecules, ${ }^{30} \mathrm{Eq}$. (1) for the minimal $E_{g}$ at LNT takes the form: $x-0.05=$ $0.49 x+1.08 \mathrm{eV}$ and gives $E_{g} \approx 2.22 \mathrm{eV}$. We obtain the same value at RT. According to our carbon NPs approach, this $E_{g}$ value can be compared with the result obtained for dry carbon NPs. Fluorescence spectra have been measured in the temperature range $77-300 \mathrm{~K}$ for the dry layers of NPs, which were produced from acetic acid. ${ }^{31}$ The existence of two energy gaps $E_{g}$ has been deduced and for the smaller one the value $E_{g}=2.197 \mathrm{eV}$ has been obtained, which is in good agreement with our result.

The theoretical $E_{g}$ value of carbon NPs can be deduced from the calculations by the density functional theory (DFT) carried out for the clusters of fused aromatic carbon rings. ${ }^{32,33}$ For a flat cluster ( $\mathrm{sp}^{2}$ hybridization) of 13 rings $E_{g} \approx 3.5 \mathrm{eV}^{32}$ and $2.5 \mathrm{eV}^{33}$ have been obtained, whereas the value $E_{g} \approx 2.2 \mathrm{eV}$ has been calculated for a more massive 37 rings cluster. ${ }^{32}$

It can be concluded from the foregoing description that the value of $E_{g}$ is determined reliably. This means that the VFS as a fluorescent marker has an operation long-wavelength edge around $575 \mathrm{~nm}$ $(2.15 \mathrm{eV})$ and cannot be used in the red and infrared spectral regions.

The invariability of the full width at half maximum (FWHM) of emission bands in the temperature range $77-295 \mathrm{~K}$ (Fig. 6) points to a weak electron-phonon coupling in the fluorescence centers. In the frames of $\mathrm{sp}^{2}$-hybridization model, this fact can be easily explained by the specificity of phonon spectra in the plane $\mathrm{sp}^{2}$ carbon lattice (graphite). According to Ref. 34, the phonon spectra of carbon dots and graphite are similar because of the analogy of the $\mathrm{sp}^{2}$ carbon lattice in both matters (the spectra of dots display more harmonics connected with the presence of oxygen). In any case, these substances possess a very high Debye temperature $\Theta$ as almost all vibration harmonics have the energies higher than $1000 \mathrm{~cm}^{-1}$. The Debye 
temperature is not determined specifically for NPs (dots) but it is known to be $2200 \mathrm{~K}$ for graphite planes formed by the $\mathrm{sp}^{2}$ orbitals. ${ }^{35}$ Thus the RT is, in effect, low temperature for carbon NPs and therefore its further lowering down to LNT does not affect electron-phonon interaction essentially. Mathematically, this can be expressed as

$$
\operatorname{FWHM}(T)=\operatorname{FWHM}(T=0 K) \sqrt{\operatorname{cth}\left(\frac{h v}{2 k T}\right)},
$$

where $h v$ is phonon energy and $k$-Boltzmann constant. In our case, the argument $h v / 2 k T(h v \geq$ $1000 \mathrm{~cm}^{-1}$ ) exceeds the value 2 even at RT. This means that the function $y=\operatorname{cth}(x)$ does not change in the range 77-300 K, and subsequently, the FWHM remains constant as it is a matter of fact in Fig. 6.

In Ref. 31, the bandwidths of the fluorescence spectra of carbon NPs in dry layers also exhibited independence on temperature changes in the interval $77-300 \mathrm{~K}$. This verification ascertains once again the validity of parallel consideration of optical phenomena in biofluids and carbon NPs.

A strong similarity between the MALDI spectra of urine fractions and GO allows us to lay a ground for the interpretation of the complicated urine spectra. A highly typical line 1 (Tables 1 and 2) corresponding to the particles with $M \approx 412 \mathrm{Da}$ can be taken as the initial link in the chain of interpretations.

There are some molecules with the appropriate masses in the human urine metabolome data base ${ }^{13}$ : stigmasterol $\mathrm{C}_{29} \mathrm{H}_{48} \mathrm{O}(M=412.69 \mathrm{Da}), 25$-Hydroxyvitamine $\mathrm{D}_{2} \mathrm{C}_{28} \mathrm{H}_{44} \mathrm{O}_{2}$ (412.65 Da) and adapalene $\mathrm{C}_{28} \mathrm{H}_{28} \mathrm{O}_{3}$ (412.52 Da). All these substances are relatively poor at oxygen $(\mathrm{C}: \mathrm{O}=29,14$ and 9.3 , respectively) and differ in this relation very much from $\mathrm{GO}$ with $\mathrm{C}: \mathrm{O}=2.5$. The factor of stoichiometry is, as it is to see below, very important for understanding the similarity of urine and GO spectra (Fig. 7). Therefore, we have looked for other metabolites and phytochemicals with the higher oxygen content. Some secondary metabolites, flavonoids and/or aglycons of glycosides, have such a higher content of oxygen. ${ }^{36}$ For instance, kaempferol $\mathrm{C}_{15} \mathrm{H}_{10} \mathrm{O}_{6}$ has the ratio $\mathrm{C}: \mathrm{O}$ just 2.5 and quercetin $\mathrm{C}_{15} \mathrm{H}_{10} \mathrm{O}_{7}-2.14$. Their masses, 286 and $302 \mathrm{Da}$ respectively, are however, far from the "needed" 412 Da. Flavonoids and glycosides are very large families of phytochemicals abundant in the human diet as food components of plant origin. ${ }^{36}$ It is not excluded that there are some unidentified chemicals, and therefore, we failed to find appropriate representatives in the data bases used. $^{13,36}$

We were compelled to create a possible new model of such a Core particle (molecule) starting not from the real-known metabolites but from GO taking into account its important properties. The GO structures are rather variable and rich for combinations of the carbon-oxygen bonds (see Sec. 2) and the C:O stoichiometry of GO can be changed in the wide range of $2.1-2.7 .{ }^{24,37,38}$ The ratio $\mathrm{C}: \mathrm{O}=2.5$ in our $\mathrm{GO}$ can be considered as an average value of different ratios $\mathrm{C}: \mathrm{O}$ in different fragments of the integral GO lattice.

Using the Avogadro software, we have composed the atomic models for some particles detected in the MALDI measurements in the range $M / Z=400$ $460 \mathrm{Da}$ (Fig. 7). A model of the Core particle $412 \mathrm{Da}$ is demonstrated in Fig. 9. It has the formula $\mathrm{C}_{23} \mathrm{O}_{8} \mathrm{H}_{8}$ with the ratio $\mathrm{C}: \mathrm{O}=2.875$ and GO-like structure. Oxygen is placed in the positions typical of GO: in the epoxy $(\mathrm{C}-\mathrm{O}-\mathrm{C})$ - atom O8, carbonyl $\mathrm{C}=\mathrm{O}(\mathrm{O} 2, \mathrm{O} 5, \mathrm{O} 6$ and $\mathrm{O} 7)$ and hydroxyl $-\mathrm{OH}(\mathrm{O} 3$ and $\mathrm{O} 4)$ groups. One oxygen atom (O1) is placed in the six-member ring corner. The largest lateral dimension of Core is $1.06 \mathrm{~nm}$ and the thickness approaches $0.25 \mathrm{~nm}$. The chemical formulae for the Core and other particles, their masses, C:O ratios, composition, MALDI lines nomenclature and characteristics are given in Table 3. All available GO and urine mass spectra data were taken into account.

One can see certain correlations between the C:O stoichiometry and characteristics of the MALDI spectra: besides the Core-related line 1, stable and typical are these which are induced by the particles of the $\mathrm{C}: \mathrm{O}$ stoichiometry 2.5 or near this value. Namely, these are the particles $\mathrm{C}_{23} \mathrm{O}_{9} \mathrm{H}_{8}$ and $\mathrm{C}_{24} \mathrm{O}_{9} \mathrm{H}_{8}$ with one additional oxygen atom or $\mathrm{CO}(\mathrm{H})$ group (lines 3 and 5) with respect to the Core particle. It seems that the stoichiometric deviation can be still rather large and this does not prevent the existence of stable MALDI particles $\left(\mathrm{C}_{23} \mathrm{O}_{8} \mathrm{H}_{8} ; 2.875\right.$ or $\left.\mathrm{C}_{24} \mathrm{O}_{10} \mathrm{H}_{8} ; 2.4\right)$. If stoichiometry deviates even more $(\mathrm{C}: \mathrm{O} \geq 3)$, the according lines are weak and rare as these are the line 2 for the particle $\mathrm{C}_{24} \mathrm{O}_{8} \mathrm{H}_{8}$ with $\mathrm{C}: \mathrm{O}=3$ and line 4 for $\mathrm{C}_{25} \mathrm{O}_{8} \mathrm{H}_{8}(\mathrm{C}: \mathrm{O}=3.125)$.

The intrinsic tensions in the model configurations from Table 3 are found to be low for the Core (1) 


\section{A. I. Kuznetsov et al.}

Table 3. Formulae, masses, $\mathrm{C} / \mathrm{O}$ ratios, composition, designations and characteristics for twinned (GO $\leftrightarrow$ urine) MALDI particles.

\begin{tabular}{lcclcl}
\hline Formulae & Mass, Da & C:O & Composition & MALDI lines & \multicolumn{1}{c}{ Line characteristics } \\
\hline $\mathrm{C}_{23} \mathrm{O}_{8} \mathrm{H}_{8}$ & 412 & 2.875 & Core & 1 & Intense, highly typical \\
$\mathrm{C}_{24} \mathrm{O}_{8} \mathrm{H}_{8}$ & 424 & 3 & Core $+\mathrm{C}$ & 2 & Weak, rare \\
$\mathrm{C}_{23} \mathrm{O}_{9} \mathrm{H}_{8}$ & 428 & 2.556 & Core $+\mathrm{O}$ & 3 & Highly typical, most prominent in urine spectra \\
$\mathrm{C}_{25} \mathrm{O}_{8} \mathrm{H}_{8}$ & 436 & 3.125 & Core $+2 \mathrm{C}$ & 4 & Weak, rare \\
$\mathrm{C}_{24} \mathrm{O}_{9} \mathrm{H}_{8}$ & 440 & 2.667 & Core $+\mathrm{CO}$ & 5 & Stable presence in GO \\
$\mathrm{C}_{24} \mathrm{O}_{10} \mathrm{H}_{8}$ & 456 & 2.4 & Core $+\mathrm{COO}$ & 6 & and urine spectra \\
\end{tabular}

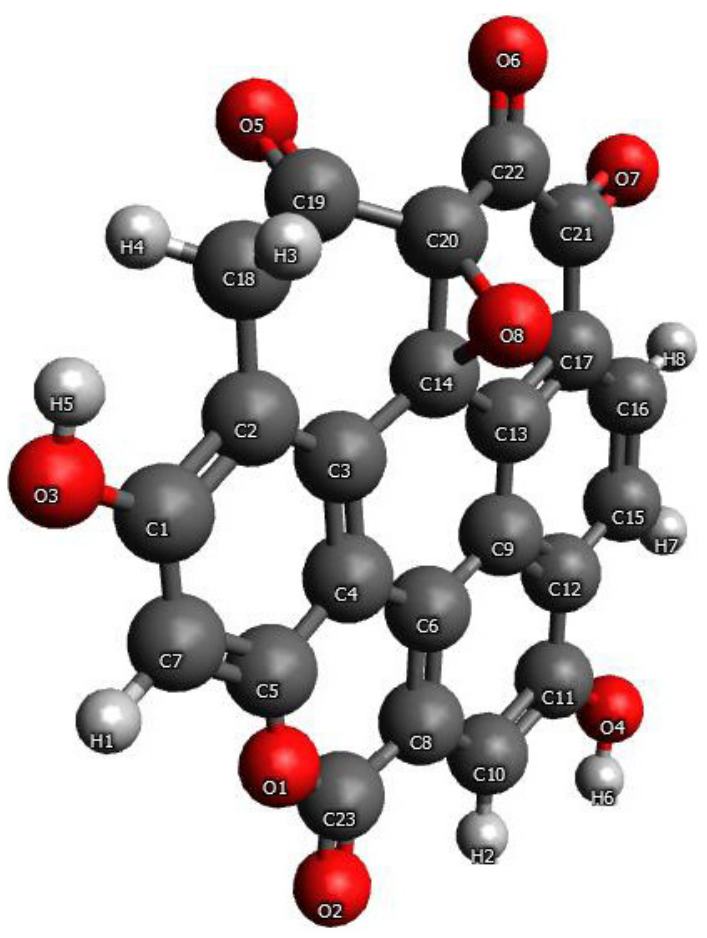

Fig. 9. A possible model of a MALDI core particle $\mathrm{C}_{23} \mathrm{O}_{8} \mathrm{H}_{8}$ with numbered atoms of carbon $\mathrm{C}$, oxygen $\mathrm{O}$ and hydrogen $\mathrm{H}$.

and stoichiometric particles $(3,5)$. For other combinations the tensions can be higher. This fact explains a lower abundance of such particles due to their eventual instability.

Five other models for the particles derived from the Core as it is characterized in Table 3 are represented in ESI, Figs. 3(a)-3(e). We demonstrate also two models of small carbon-oxygen-hydrogen NPs consisting of three planar particles $\mathrm{C}_{23} \mathrm{O}_{8} \mathrm{H}_{8}$ with no epoxy oxygen atom (ESI, Fig. 4(a)) and with one and two epoxy $\mathrm{O}$ atoms at marginal layers [ESI, Fig. 4(b)]. Evidently, one can construct other Core models and according derivatives with different geometries and slightly deviating compositions. This is the matter of further modeling and verifications which are being underway.

It is possible to find a compliance between our model particles and the mass distribution in the chromatograms of urine. ${ }^{8,10}$ For instance, there are two peaks in the chromatograms ${ }^{8}$ at 500 and 1250 Da which can be related to the Core and other single particles and to the triple complex in ESI, Figs. 4(a) and 4(b), respectively. Qualitatively, the same chromatogram picture has been obtained in Ref. 10, but the masses are not given precisely there.

To our knowledge, it is for the first time that the nitrogen-free particles (molecules) are observed in the mass spectra of GO in parallel to the 
observation of similar particles in the urine spectra. This result strongly points to the availability of nitrogen-free substances in biofluids and demonstrates the possibility to observe them in the MALDI mass spectra (compare, e.g., Ref. 39). At the same time, it is a challenging fact that one cannot attribute the most typical and intense lines in the MALDI spectra to the known primary or secondary metabolites, in spite of the rich available databases ${ }^{13,36}$ containing over 45,000 items in total.

Therefore, we can suppose the existence of particles with the composition and structure similar to GO, arising in the body by specific reactions. In vitro synthesis of $\mathrm{GO}$ using glucose as a sole reagent was carried out earlier. ${ }^{40}$ The GO films were obtained by heating aqueous glucose solutions for $70-660 \mathrm{~min}$ at $433-493 \mathrm{~K}$. The heat was the only affecting factor in this synthesis. It can be assumed that similar reactions may occur also in human body at $T \approx 310 \mathrm{~K}$ with a lower rate. If the rate of these reactions depends exponentially on the temperature according to the law $\exp (-\Delta E / k T)$, where $\Delta E$ is the activation energy of the process, then by the values of $(\Delta E / k T) \leq 1$ the rate of reactions at 435 and $310 \mathrm{~K}$ will differ only by the factor of 1.5 or less.

The synthesis transition from glucose to GO may be compared to the reactions which give rise to AGEs. The related browning Maillard reaction and the emergence of AGEs occur during cooking (in vitro) at high speed, but "occurs over period of weeks" ${ }^{28}$ in vivo. The large starting role of endogenous sugars (glucose and other modifications) in the reactions of both kinds can provide such a parallel emergence of AGEs and GO-related NPs in the body.

An acute question has to be answered. Are graphene and GO safe or toxic? ${ }^{38,41}$ We have carried out the following pilot experiment. A urine fraction, practically the same as that used in this study but diluted, has been tried as an agent for endocytosis by HeLa cells in vitro. A massive and prompt death of cells has been observed, ${ }^{42}$ which points to a potential biological danger of usual human urine. We hope that further investigations allow enlarging the nomenclature of toxins and enriching the AGEs list by nitrogen-free, GO-related ultrasmall NPs with the projection onto the high mortality problem among the ESRD patients.

\section{Conclusions}

The development of a NP approach to elucidate the optical properties of biofluids, especially the VFS, has been continued with the extensive use of solid state phases. The examination carried out with a SEM shows that metabolites of protein-free biofluid fractions (urine, serum) form on a substrate the carbon-oxygen NPs of diameter $5 \sim 100 \mathrm{~nm}$, which contain also nitrogen in moderate concentrations (8-9wt.\%). Initial existence of small-sized $(d \leq$ $5 \mathrm{~nm})$ NPs of the same nature in the liquid medium of biofluids was deduced by taking into account also the previous results on SAXS and DLS.

The fluorescence of the dry (lyophilized) samples of urine shows the dependence of emission spectra on excitation energy (bathochromic or red shift), which can be easily observed with a confocal microscope together with existence of excitation of threshold energy $E_{g} \approx 2.15 \mathrm{eV}$ and in fluorescence measurements at various temperatures in the range 77-295 K. The bathochromic shift can be described by linear equations in an energy scale at different temperatures that allows making an estimation of the minimal energy gap $E_{g}$ of the VFS: $E_{g} \approx 2.1-$ $2.15 \mathrm{eV}$. The $E_{g}$ value determines the VFS as a fluorescent marker for working in the visible but not in the infrared region.

Comparative MALDI experiments with the use of GO and urine fractions have revealed a strong similarity in their mass spectra in the range of $M / Z=400-460 \mathrm{Da}$. A consistent interpretation is given on the basis of the existence of a highly typical particle with the mass $412 \mathrm{Da}$ (called Core and designated as $\mathrm{C}_{23} \mathrm{O}_{8} \mathrm{H}_{8}$ ) and its derivatives as a result of the additional inclusion of the $\mathrm{O}$ atoms or $\mathrm{OH},-\mathrm{COH}$ and $-\mathrm{COOH}$ groups. Spatially stable models for these MALDI particles were composed. These particles can be considered also as fragments of larger complexes or NPs, possibly, with masses in the range around $1 \mathrm{kDa}$, typical for the VFS in all biofluids studied.

As the main result, one can consider the observation of two types of particles in biofluids: nitrogen containing carbon-oxygen NPs (SEM images and elemental analysis in situ) and nitrogen-free carbon-oxygen-hydrogen particles (MALDI mass spectra). The former group of NPs can with high probability belong to the family of AGEs, whereas the nitrogen-free particles can be closely related to fluorescent GO structures. Biochemical origin of 
those still remains to be revealed. The strong analogy of the particles derived from GO and human urine was observed, to our knowledge, for the first time. It opens the possibility to add to the AGEs toxins also the GO-related particles and to tackle the problem of high mortality among ESRD patients on a more solid basis.

At present, there is arising critics about evaluation methods of hemodialysis efficacy performed as monitoring of excretion of one definite solute (metabolite) which is usually used to be urea (see, e.g., Ref. 43). Representatives of the European Uremic Toxin Work Group state that "... the uremic milieu is complex, and solutes may affect various biological systems, possibly in a synergetic manner". ${ }^{44}$ We can assume that our consideration of endogenous metabolites in biofluids as heteronuclear NPs could serve as such an integrative and synergetic approach.

It is well known that fluorescence gives much information not only about the fluorescence center itself but also on its atomic environment as does, e.g., amino acid tryptophan in proteins. ${ }^{30}$ Therefore, one can expect that auto-fluorescence of VFS is a more informative synergetic signal about "uremic milieu" in ESRD patients' biofluids than the concentration of some separate metabolite (urea, creatinine, indoxyl sulfate, etc). We can believe that our first observations of correlation "intensity of VF in hemodialysates-ESRD patients' mortality" will be confirmed.

Additional control experiments with cells on biological activity of waste products containing in biofluids and taken in parallel from the ESRD patients and healthy controls should be continued.

\section{Acknowledgments}

We acknowledge the financial support for this work by the Archimedes project 3.2.1101.12-0011. This work was supported in part by the institutional research funding IUT (IUT 2-26) of the Estonian Ministry of Education and Research. The author A. K. thanks Prof. V. Tuchin for stimulating attention and valuable support of this work. The authors thank Profs. V. Sammelselg and U. Soomets, Drs. M. Marandi and A. Ottas for providing the possibility and valuable help to carry out the experiments at the SEM and MALDI unites, R. Kurg for the possibility to use a confocal microscope, Dr. M. Jayanta for providing GO samples as well as Tartu University Hospital dialysis division nurses for kind collaboration in collecting hemodialysis fluid samples.

\section{Ethics}

The study has been approved by the Ethics Committee on Human Research of the Tartu University, Estonia (protocol no 219/M-19; 2012).

\section{References}

1. H. A. Schwertner, "Isolation and chromatographic analysis of unidentified fluorescence in biological fluids of patients with chronic renal disease," Nephron 31, 209-211 (1982).

2. G. Münch, R. Keis, A. Wessels, P. Riederer, U. Bahner, A. Heidland, T. Niwa, H.-D. Lemke and R. Schinzel, "Determination of advanced glycation end products in serum by fluorescence spectroscopy and competitive ELISA," Eur. J. Clin. Chem. Clin. Biochem. 35, 669-677 (1997).

3. A. Kuznetsov, A. Frorip, M. Ots-Rosenberg, A. Sünter, "Blue and UV fluorescence of biological fluids and carbon nanodots," Proc. of SPIE. 9032, 90320C1-90320C11 (2013).

4. A. Kuznetsov, A. Frorip, A. Maiste, M. OtsRosenberg, A. Sünter, "Visible auto-fluorescence in biological fluids as biomarker of pathological processes and new monitoring tool," J. Innov. Opt. Health Sci. 8(3), 1541003-1-1541003-9 (2014), doi: 10.1142/S1793545815410035.

5. P. J. Tornalley, "Advanced glycation end products in renal failure," J. Renal Nutr. 16, 178-184 (2006).

6. A. Kuznetsov, A. Frorip, A. Maiste, M. OtsRosenberg, A. Sünter, J. Sablonin, J. Vasil'chenko, "Advanced glycation end products in hemodialysates as fluorescent and optical absorption markers of patients mortality," Proc. SPIE 9421, Eighth Int. Conf. Advanced Optical Materials and Devices, 94210L, doi: 10.1117/12.2083585.

7. D. Fuentealba, B. Friguel, E. Silva, "Advanced glycation endproducts induce photocrosslinking and oxidation of bovine lens proteins through type-I mechanism," Photochem. Photobiol. 85, 185-194 (2009).

8. K. Yanagisawa, Z. Makita, K. Shiroshita, T. Ueda, T. Fusegawa, S. Kuwajima, M. Takeuchi, T. Koike, "Specific fluorescence assay for advanced glycation end products in blood and urine of diabetic patients," Metabolism 47, 1348-1353 (1998).

9. P. Papanastasiou, L. Gras, H. Rodela, A. Patrikarea, D. Oreopulos, E. P. Diamandis, "Immunological 
guantification of advanced glycosylation end-products in the serum of patients on hemodialysis or CAPD," Kidney Int. 46, 216-222 (1994).

10. R. Dolhofer-Bliesener, B. Lechner, R. Deppisch, E. Ritz, K. D. Gerbitz, "Immunological determination of advanced glycosylation end-products in human blood and urine," Nephrol. Dial. Transplant. 10, 657-664 (1995).

11. M. A. Friedlander, Y. C. Wu, A. Elgawish, V. M. Monnier, "Early and advanced glycosylation end products," J. Clin. Invest. 97, 728-735 (1996).

12. M. Takeuchi, Z. Makita, K. Yanagisawa, Y. Kameda, T. Koike, "Detection of Noncarboxymethyllysine and Carboxymethyllysine advanced glycation end products (AGE) in serum of diabetic patients," Mol. Med. 5, 393-405 (1999).

13. www.hmdb.ca.

14. A. Kuznetsov, A. Frorip, M. Ots-Rosenberg, A. Sünter, S. Patsaeva, "Endogenous carbon nanoparticles as source of blue autofluorescence in biological fluids: Eventual use in clinical praxis," Saratov Fall Meeting 2013, Biophotonics, Internet Invited Lecture, 25-28 September 2013, Available at http://sfm.eventry.org/report/911.

15. A. C. Raff, T. W. Meyer, T. H. Hostetter, "New insights into uremic toxicity," Curr. Opin. Nephrol. Hypertens. 17, 560-565 (2008).

16. M. E. Elsharif, "Mortality rate of patients with end stage renal disease on regular hemodialysis: A single center study," Saudi J. Kidney Dis. Transpl. 22, 594-596 (2011).

17. S. B. Schwedler, Th. Metzger, R. Schinzel, Ch. Wanner, "Advanced glycation end products and mortality in hemodialysis patients," Kidney Int. 62, 301-310 (2002).

18. W. F. Owen, Jr., N. L. Lew, Y. Liu, E. G. Lowrie, J. M. Lasarus, "The urea reduction ratio and serum albumin concentration as predictors of mortality in patients undergoing hemodialysis," New Engl. J. Med. 329, 1001-1006 (1993).

19. J. Holmar, I. Fridolin, F. Uhlin, A. Fernström, M. Luman, "Estimation of dialysis patients' survival through combined approach of small molecule uremic markers," Proc. Estonian Acad. Sci. 63, 315321 (2014).

20. A. Dawnay, "Renal clearance of glycation adducts: Anti-glycation defence in uraemia and dialysis," Biochem. Soc. Trans. 31, 1386-1389 (2003).

21. L. Ratke, P. W. Voorhees, Growth and Coarsening: Ostwald Ripening in Material Processing, Springer Verlag, Berlin-Heidelberg, New York (2002).

22. H. Li, Z. Kang, Y. Liu and S.-T. Lee, Carbon nanodots: Synthesis, properties and applications," J. Mater. Chem. 12, 24230-24253 (2012).
23. D. Pan, J. Zhang, Z. Li, M. Wu, "Hydrothermal route for cutting graphene sheets into blue-luminescent graphene quantum dots," Adv. Mater. 22, 734-738 (2010).

24. J. Shang, L. Ma, J. Li, W. Ai, T. Yu, G. G. Gurzadyan, "The origin of fluorescence from graphene oxide," Sci. Rep. 2 (2012), doi: 10.1038/srep00792.

25. F. C. Shi, J. Menga, C. Deng, "Facile synthesis of magnetic graphene and carbon nanotube composites as a novel matrix and adsorbent for enrichment and detection of small molecules by MALDI-TOF MS," J. Mater. Chem. 22, 20778-20785 (2012), doi: 10.1039/C2JM34745H.

26. A. Kuznetsov, A. Frorip, I. Kudrjavtseva, V. Nagirny, M. Ots-Rosenberg, I. Romet, A. Sünter, J. Vassil'chenko, E-MRS 2013 Fall Meeting, Conference Program, G.VI 12 Symp G, http://www.emrsstrasbourg.com/index.php?option $=$ com_abstract \& task $=$ view $\&$ id $=228 \&$ day $=2013-0919 \&$ year $=2013$ \&Itemid=99999999\&id_season $=10$.

27. Y.-M. Long, Ch.-H. Zhou, Zh.-L. Zhang, Zh.-Q. Tian, L. Bao, Y. Lin, D.-W. Pang, "Shifting and non-shifting fluorescence emitted by carbon nanodots," J. Mater. Chem. 22, 5917-5920 (2012).

28. R. Singh, A. Barden, T. Mori, L. Beilin, "Advanced glycation end-products: A review," Diabetologia 44, 129-146 (2001).

29. http://en.wikipedia.org/wiki/Amadori_rearr angement.

30. J. R. Lakowicz, Principles of Fluorescence Spectroscopy, Springer, New York (2006), pp. 63-88.

31. P. Yu, X. Wen, Y.-R. Toh and J. Tang, "Temperature dependent fluorescence in carbon dots," J. Phys. Chem. 116, 25552-25557 (2012).

32. G. Eda, Y.-Y. Lin, C. Mattevi, H. Yamaguchi, H.-An Chen, I-Sh. Chen, Ch.-W. Chen, M. Chhowalla, "Blue photoluminescence from chemically derived graphene oxide," Adv. Mater. 22, 505-509 (2010).

33. S. H. Jin, D. H. Kim, G. H. Jun, S. H. Hong, S. Jeon, "Tuning the photoluminescence of graphene quantum dots through the charge transfer effect of functional groups," ACS NANO 7, 1239-1245 (2013).

34. H. Ming, Z. Ma, Y. Liu, K. Pan, H. Yu, F. Wang, Z. Kang, "Large scale electrochemical synthesis of high quality carbon nanodots and their photocatalytic property," Dalton Trans. 41, 9526-9531 (2012).

35. B. T. Kelly, P. L. Walker, Jr, "Theory of thermal expansion of a graphite crystal in the semi-continuum model," Carbon 8, 211-226 (1970).

36. USDA Database for the Flavonoid Content of Selected Foods, Release 3.1, Prepared by Seema Bhagwat, David B. Haytowitz and Joanne M. 
Holden (ret.), Available at http://www.ars.usda. gov/SP2UserFiles/Place/80400525/Data/Flav/ Flav3-1.pdf.

37. D. R. Dreyer, S. Park, Ch. W. Bielawski, R. S. Ruoff, "The chemistry of graphene oxide," Chem. Soc. Rev. 39, 228-240 (2010).

38. A. Bianco, "Graphene: Safe or toxic? The two faces of the medal," Angew. Chem. Int. Ed. 52, 4986-4997 (2013).

39. J. Zaia, "Mass spectrometry and glycomics," $J$. Integr. Biol. 14, 401-418 (2010).

40. L. Tang, X. Li, R. Ji, K. S. Teng, G. Tai, J. Ye, C. Wei, S. P. Lau, "Bottom-up synthesis of large-scale graphene oxide nanosheets," J. Mater. Chem. 22, 5676-5683 (2012).

41. V. C. Sanchez, A. Jachak, R. H. Hurt, A. B. Kane, "Biological interactions of graphene-family nanomaterials - An interdisciplinary review," Chem. Res. Toxicol. 25, 15-34 (2012), doi: 10.1021/tx200339h.
42. A. Kuznetsov, A. Frorip, J. Kozlova, M. Pooga, M. Ots-Rosenberg, A. Sünter, "Visible auto-fluorescence substance in biological fluids: Source or outcome of pathological processes?" The 2nd Int. Conf. Bioinspired and Biobased Chemistry\&Materials, 15-17 October 2014, Nice, http://sites.unice.fr/ site/tarrade/conferencesnice2014/downloads/FullProgram.pdf.

43. C. Basile, C. Lomonte, "Kt/V urea does not tell it all," Nephrol. Dial. Transplant. 0, 1-4 (2012), doi: 10.1093/ndt/gfr786.

44. F. Duranton, G. Cohen, R. De Smet, M. Rodrigues, J. Jankowski, R. Vanholder, A. Argiles, "Normal and pathologic concentration of uremic toxins," $J$. Am. Soc. Nephrol. 23, 1258-1270 (2012). 